\title{
ON THE DYNAMIC SNAP-THROUGH OF A SHALLOW CYLINDRICAL SHELL SUBJECT TO NEARLY SYMMETRIC IMPULSIVE LOADING
}

\author{
L. J. OvenshiRE and I. K. MCIvor \\ The University of Michigan, Ann Arbor
}

\begin{abstract}
Shallow shell theory is used to investigate the nonlinear response of a cylindrical panel undergoing plane motion in response to nearly symmetric impulsive pressure imparting an initial velocity distribution to the shell. A sufficient condition for stability is obtained by considering the critical or equilibrium configurations. For a cosine distribution the actual critical value to produce immediate dynamic snapthrough is obtained. For most geometries the sufficiency condition is a very conservative estimate of the critical value. The critical value is sensitive to small deviations from symmetry. The shell may also exhibit a delayed snap-through at significantly lower values of initial velocity. In both cases, however. the sufficiency condition remains a conservative estimate. It is inferred from a qualitative topological argument that markedly different results can be expected for other distributions
\end{abstract}

\section{INTRODUCTION}

THE problem of the dynamic snap-through of slightly curved elements subject to time dependent loads has generated an extensive literature. Of particular interest in the present paper is the case of impulsive loading. If the duration of the load is sufficiently short. its effect is to impart a velocity distribution to the structure. Thus we are concerned with free motion due to a prescribed initial velocity distribution. References pertinent to the study may be found in Hsu [1] and McIvor and Popelar [2].

The physical essence of instability is that small changes in prescribed conditions result in markedly different responses. Typically, snap-through under impulsive loads has been defined as a very rapid or discontinuous change in an appropriate response parameter with respect to small changes in the magnitude of the initial velocity. The value of the velocity when such a discontinuity occurs is taken as the critical value. Although this approach is attractive in that it yields physically meaningful results, it generally requires extensive integration of the equations of motion for each particular problem.

A more general approach has been presented by Hsu [1]. He has formulated a theory of dynamic stability of elastic bodies subject to prescribed initial conditions based on consideration of trajectories in a functional phase space. The critical or equilibrium configurations play a dominant role in the theory. He obtains a sufficient condition for stability which states that the point corresponding to the prescribed initial conditions must be located in the region of phase space surrounding the stable equilibrium configuration under consideration and within the energy surface passing through the "nearest" critical configuration. For the problem of impulsive loading of an initially undeformed body, this is equivalent to the statement that the total energy initially imparted to the body is less than the potential energy associated with the "nearest" critical configuration. The "nearest" configuration is the first critical point encountered by successive energy surfaces expanding 
about the equilibrium state of interest. It is necessarily an unstable equilibrium configuration. As an example of the theory, the exact sufficiency condition for a sinusoidal arch was obtained. More recently in a series of papers [3-5], the theory has been used to extensively investigate the behavior of shallow arches.

For a given distribution of initial velocity, violation of the sufficiency condition does not in general imply dynamic snap-through. Thus, as most recently pointed out by Huang and Nachbar [6], it is still necessary to integrate the equations of motion to obtain a stability criterion for a particular problem. In general this is not only a more difficult problem, but also the integration must be repeated for every different distribution. It is if interest, then, to compare results obtained from integrating the equations of motion with the sufficiency condition to ascertain under what conditions it will provide a reasonable estimate of the actual initial velocity to produce snap-through.

The present paper employs shallow shell theory to investigate the nonlinear, plane motion of a simply supported cylindrical panel subject to nearly symmetric initial velocity distributions. Although formulated for a long cylindrical panel, in dimensionless form the equations are applicable to the circular arch. Following Hsu, the sufficient condition for stability is obtained by determining the critical configurations of the shell. The results are qualitatively similar to those obtained by $\mathrm{Hsu}$, but the use of shallow shell equations rather than curved beam equations leads to small quantitative differences.

The actual nonlinear response for a cosine initial velocity distribution is obtained. The radial displacement is expanded in a series of linear vibrational mode shapes. Galerkin : method reduces the governing equations to a system of ordinary differential equations which are numerically integrated. The number of modes retained was determined by numerical convergence studies and was found to depend upon the shell geometry. The analysis is similar to that employed by Mclvor and Popelar [2], but the two mode solution used there was found to be inaccurate for the entire range of geometries considered.

In general the one or two degree of freedom systems employed by most previous investigators do not appear to be adequate for obtaining a quantitative criterion by direct integration.

It is found that for most geometries the sufficiency condition is a highly conservative estimate of the initial velocity required for immediate dynamic snap-through. It is shown. however, that the critical velocity is sensitive to small deviations from symmetry. The shell may also exhibit a delayed snap-through at significantly lower values. In both cases the sufficiency condition remains a conservative estimate.

A qualitative topological argument is given to interpret the variety of responses obtained. It is inferred from this argument that no general conclusions can be drawn from the results for the cosine distribution. Other symmetric distributions may give very different results. $A$ stability criterion obtained by integrating the equations of motion is strongly dependent upon the actual loading distribution. Although in many cases the sufficiency condition is unduly conservative, it does provide a bound valid for all distributions.

\section{FORMULATION}

The shell geometry is shown in Fig. 1. We consider plane motion of the shell. Before deformation a midsurface point $P$ has polar coordinates $(a, \theta)$. The point in the deformed shell is located by the displacement vector

$$
\delta=a \dot{\psi} \mathbf{t}+a \grave{n} \mathbf{n}
$$




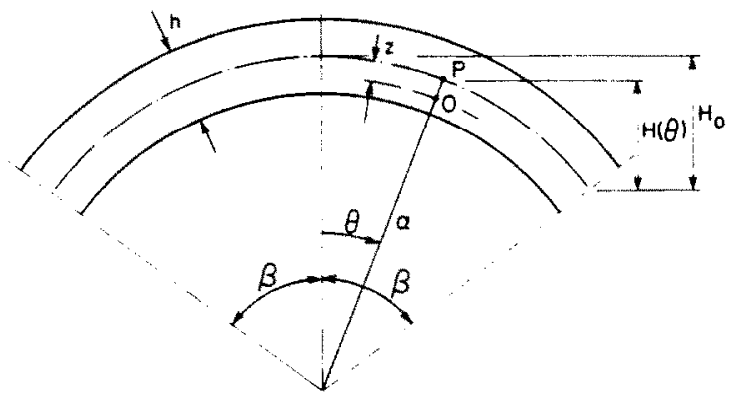

Fig. 1. Shell geometry.

where $a$ is the radius of the cylinder and $\mathbf{t}$ and $\mathbf{n}$ are the unit tangent and normal vectors to the midsurface of the undeformed shell. Thus $\zeta$ and $\psi$ are dimensionless radial and tangential displacement components.

With the usual assumptions of thin shell theory; the tangential strain is

$$
\varepsilon=\bar{N}+(z / a) \bar{M}
$$

where

$$
\begin{aligned}
& \bar{N}=\psi^{\prime}-\zeta+\frac{1}{2} \zeta^{\prime 2} \\
& \bar{M}=-(\zeta)+\zeta)
\end{aligned}
$$

and $z$ is the radial distance below the undeformed midsurface. The prime denotes differentiation with respect to $\theta$. The quantities $\bar{N}$ and $\bar{M}$ are the midsurface strain and change in curvature respectively. In the nonlinear term in (3) we have neglected $\psi$ in comparison to $\zeta$.

Letting $N$ denote the membrane force and $M$ the bending moment per unit length of cylinder, the constitutive equations are

$$
\begin{aligned}
& N=\frac{E h}{1-v^{2}} \bar{N} \\
& M=\frac{E h^{3}}{12\left(1-v^{2}\right) a} \bar{M}
\end{aligned}
$$

where $h$ is the shell thickness, $E$ is Young's modulus and $v$ is Poisson's ratio.

We introduce dimensionless kinetic and strain energies as

$$
\begin{aligned}
& \bar{T}=\frac{T\left(1-v^{2}\right)}{E a h}=\frac{1}{2} \int_{-\beta}^{\beta}\left(\dot{\zeta}^{2}+\dot{\psi}^{2}\right) \mathrm{d} \theta \\
& \bar{V}=\frac{V\left(1-v^{2}\right)}{E a h}=\frac{1}{2} \int_{-\beta}^{\beta}\left(\bar{N}^{2}+\alpha^{2} \bar{M}^{2}\right) \mathrm{d} \theta
\end{aligned}
$$

in which $T$ and $V$ are the kinetic and strain energies per unit length of cylinder and

$$
\alpha^{2}=\frac{1}{12}(h / a)^{2}
$$


The dot denotes differentiation with respect to a dimensionless time $t$, where

$$
\begin{aligned}
\tau & =c t a \\
c^{2} & =E / \rho\left(1-r^{2}\right)
\end{aligned}
$$

in which $t$ is real time and $\rho$ is the mass density.

The equations of motion are obtained through Hamilton's principle. For free vibration they are

$$
\begin{aligned}
& \ddot{B}-x^{2}\left(\bar{M}^{\prime \prime}+\bar{M}\right)-\bar{N}-\left(\bar{N}_{\zeta}^{\prime \prime}\right)^{\prime}=0 \\
& \psi-\bar{N}^{\prime}=0 .
\end{aligned}
$$

The natural boundary conditions also follow from Hamilton's principle. For the simple support conditions of interest here. they are

$$
\begin{aligned}
& \vdots \pm \beta, \tau)=0 \\
& \dot{\psi}( \pm \beta, \tau)=0 \\
& \bar{\nabla}( \pm \beta, \tau)=0 .
\end{aligned}
$$

\section{SUFFICIENT CONDITION FOR STABILITY}

Following [1], we first obtain a sufficient condition for stability by determining the critical or equilibrium configurations. Setting the time derivatives to zero and using (4) and $(9 b)$, equation $(9 a)$ reduces to

$$
\varrho^{\prime \prime \prime \prime}+\left(2-\bar{N} / x^{2}\right) \zeta^{\prime \prime}+\zeta=\bar{N}: x^{2}
$$

It follows from (9b) that $\bar{N}$ is a constant. Integrating (3) and using (10b) then yields

$$
\bar{N}=-\frac{1}{2} \int_{-1}^{1}\left(s-\frac{1}{2} \omega^{2}\right) \mathrm{d} \Gamma
$$

in which

$$
\Gamma=\theta i \beta .
$$

Equations (11) and (12) together with the boundary conditions (10a) and (10c) determine the critical configurations. The trivial solution $(\zeta=\bar{N}=0)$ is the undeformed state. Depending upon the geometric parameters, there may be none, two, or more additional configurations.

The solution is elementary but algebraically somewhat lengthy. It is given in detail in Ref. [7]. The exact solution contains a number of terms that are negligible when computing numerical results. The validity of an approximate solution obtained by neglecting such terms is verified in Ref. [7]. For brevity, only the approximate form of the solution is given here. It is

$$
\check{\zeta}=\beta^{2}\left[\frac{1}{q^{2}}(b \sin q \Gamma+1-\cos q \Gamma \cos q)+\frac{1}{2}\left(1-\Gamma^{2}\right)\right]
$$


where $q$ is the eigenvalue and $b$ a constant associated with a specific configuration. The eigenvalue $q$ is related to the membrane strain through the relation

$$
\bar{N}=-\beta^{2} q^{2} / \lambda^{4}
$$

where

$$
\hat{\lambda}^{2}=\beta^{2} / \alpha=(, 48)\left(H_{0} / h\right) .
$$

There are two distinct cases. For symmetric configurations

$$
b=0
$$

and $q$ is a root of the characteristic equation

$$
-\sin 2 q+2 q\left[1-\cos ^{2} q g(q)\right]=0
$$

where

$$
\left.g(q)=\frac{2}{3} q^{2}-4(q) \lambda\right)^{4} .
$$

There may also exist nonsymmetric configurations for which

$$
\begin{aligned}
q & =n \pi \\
b^{2} & =\frac{2}{3}(n \pi)^{2}-1-4(n \pi / \lambda)^{4} \quad n=1,2, \ldots
\end{aligned}
$$

Clearly nonsymmetric configurations exist only when the right hand side of $(19 \mathrm{~b})$ is positive. Setting $b^{2}$ equal to zero and solving for $\lambda$ for a specific value of $n$ equal to $N$, we obtain

$$
\tilde{i}_{N}=\left[6 N^{4} \pi^{4} /\left(N^{2} \pi^{2}-\frac{3}{2}\right)\right]^{\frac{1}{4}}
$$

It can be shown that

$$
\begin{aligned}
& b^{2}>0 \quad \text { for } 1 \leq n \leq N \quad \text { if } \lambda>\tilde{\lambda}_{N} \\
& b^{2}<0 \quad \text { for } n \geq N \quad \text { if } i<\tilde{i}_{N} \text {. }
\end{aligned}
$$

Thus we conclude that exactly $N$ pairs $\dagger$ of nonsymmetric configurations exist for

$$
\tilde{i}_{N}<\lambda<\tilde{i}_{N+1} \text {. }
$$

No nonsymmetric configurations exist for $\lambda$ less than $\hat{\lambda}_{1}\left(\tilde{\lambda}_{1}=2.891\right)$.

To investigate the existence of symmetric configurations; we must numerically examine the roots of (18a) as a function of $\lambda$. For sufficiently small values of $\lambda$, no roots exist. As $\lambda$ increases special values, denoted as $\bar{\lambda}_{N}$, are found above which two additional roots exist. Thus exactly $N$ pairs of symmetric configurations exist for

$$
\bar{\lambda}_{N}<\lambda<\bar{i}_{N+1} \text {. }
$$

The values of $\bar{i}_{N}$ alternate with $\tilde{i}_{N}$ such that

$$
\bar{\lambda}_{N}<\tilde{\lambda}_{N}<\bar{\lambda}_{N+1}
$$

The values of $\lambda_{N}$ obtained from the numerical study of (18a) together with the values of $\tilde{i}_{N}$ obtained from (20) are shown in Fig. 2.

+ The pair arises. of course, from taking the square root to obtain $b$. One member of the pair is the mirror image of the other reflected in the ray $\theta=0$. 


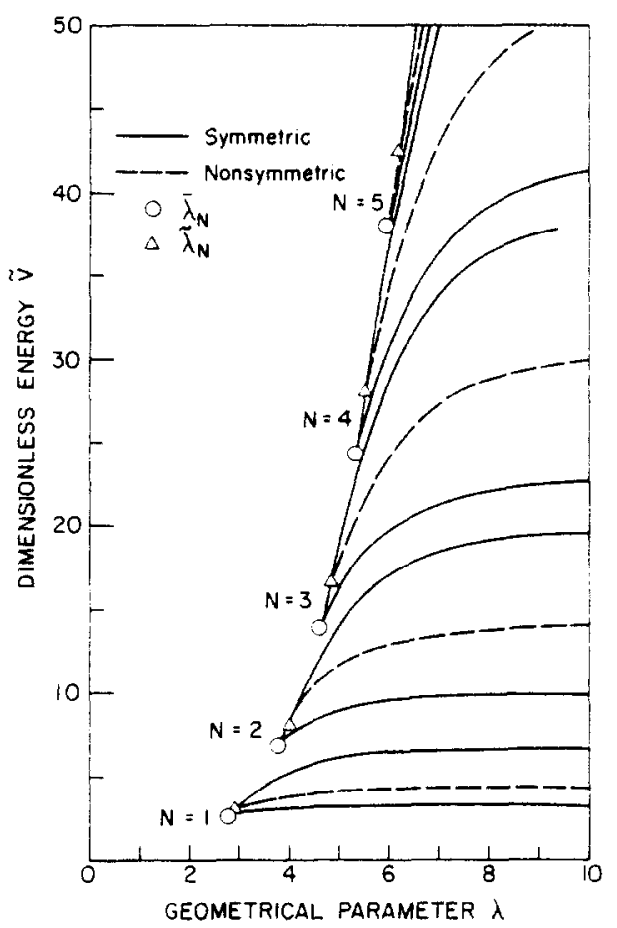

FIG. 2. Energy of critical configurations vs. geometric parameter $\lambda$.

To obtain a sufficient condition for stability. we examine the energy state associated with each critical configuration. It is convenient to introduce a second dimensionless form of the potential energy as

$$
\bar{V}=\bar{V} / \beta \alpha^{2}
$$

where $\vec{V}$ is given by (6b). We introduce $(4),(14)$ and $(15)$ into (6b) and simplify the result by using (18) for the symmetric configurations or (19) for the nonsymmetric configurations. In both cases we obtain

$$
\tilde{V}=1+\frac{1}{3} q^{2}-(q / \lambda)^{4}-\tan q i q .
$$

Plots of $\tilde{V}$ vs. $\lambda$ are shown in Fig. 2. The distribution of critical configurations as a function of $\lambda$ is evident in the figure.

We can now state a sufficient condition for stability under impulsive loads. As shown by Hsu [1] an initially undeformed elastic body subject to an initial velocity distribution is dynamically stable if the energy imparted to the body is less than the potential energy associated with the "nearest" unstable critical configuration. In the present problem the nontrivial configuration with the lowest energy is a stable symmetric configuration. Thus the energy associated with the "nearest" unstable critical configuration cannot be less than the second lowest energy curve. We denote this energy level as $\vec{V}_{c}$. It is plotted in Fig. 3 to a more convenient scale. For $\lambda$ less than $\lambda_{1}$ there are no critical configurations, and the shell cannot snap-through. For the narrow range of $\dot{\lambda}$ between $\bar{\lambda}_{1}$ and $\tilde{\lambda}_{1}$, the sufficiency condition is

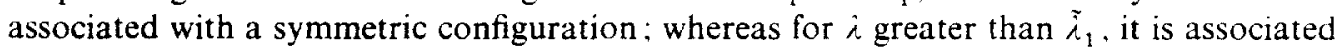
with a nonsymmetric configuration. 


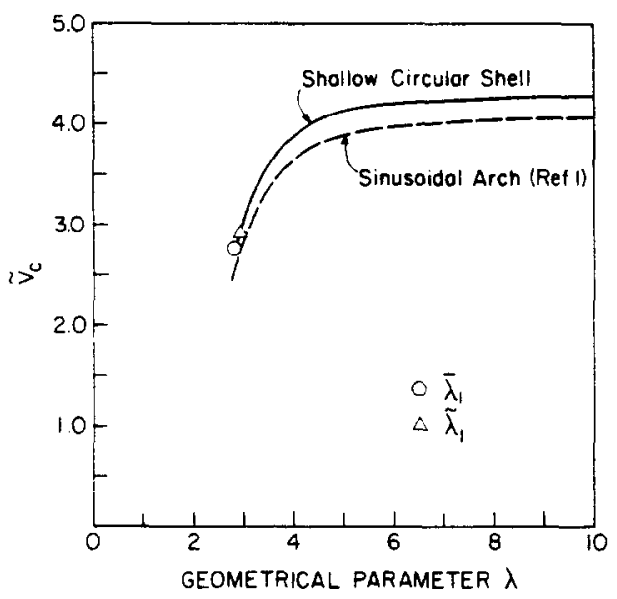

FIG. 3. Sufficiency condition.

The sufficiency condition obtained by Hsu for a sinusoidal arch using curved beam equations is also shown in Fig. 3. The two results are qualitatively the same. but exhibit small quantitative differences.

\section{NONLINEAR RESPONSE TO IMPULSIVE PRESSURE}

To investigate the actual response to impulsive pressure, we seek solutions of the nonlinear equations of motion. Introducing (4) into (9a) yields

$$
\ddot{\zeta}+\alpha^{2}\left(\zeta^{\prime \prime \prime \prime}+2 \zeta^{\prime \prime}+\zeta\right)-\bar{N}-\left(\bar{N} \zeta^{\prime \prime}\right)=0
$$

Reissner [8] has shown that tangential inertia may be neglected in the linear response of shallow shells. Assuming that this holds for the somewhat larger motions considered here, it follows from (9b) that $\bar{N}$ is spatially constant. Thus $\bar{N}$ may still be represented by the integral expression (15), i.e.

$$
\cdot \bar{N}(t)=-\frac{1}{2} \int_{-1}^{1}\left(\zeta-\frac{1}{2} \zeta^{\prime 2}\right) d \Gamma
$$

We represent the radial displacement by the series

$$
\zeta=\beta^{2} \sum_{m} T_{m}(\tau) Z_{m}(\theta)
$$

where the factor $\beta^{2}$ has been introduced for convenienca The quantities $T_{m}$ are time varying coefficients and $Z_{m}$ are the set of linear mode shapes. The complete set consists of a set of symmetric and a set of asymmetric modes. When necessary to distinguish between them, we will denote the symmetric modes by $\tilde{Z}_{m}$ and the asymmetric modes by $\bar{Z}_{m}$. The mode shapes have been determined previously in Ref. [7] and are listed in the Appendix for convenience. 
An infinite set of ordinary differential equations in the coefficients are now obtained through Galerkin's method. This set is

$$
\ddot{T}_{m}+\mu_{m}^{2} T_{m}+\frac{1}{2} \sum_{i} \sum_{j}\left(n_{m} \varepsilon_{i j}+2 n_{i} \varepsilon_{m j}+\frac{1}{2} \varepsilon_{i j} \sum_{k} \varepsilon_{m k} T_{k}\right) T_{i} T_{j}=0 .
$$

When a subscript $m$ is associated with a symmetric mode, we have

$$
\begin{aligned}
& \mu_{m}=\tilde{\mu}_{m} \\
& n_{m}=\left(2 A_{m} u_{m}^{2} v^{2}{ }_{m} / \lambda^{4}\right) \cos v_{m}
\end{aligned}
$$

where $\tilde{\mu}_{m}, A_{m}, u_{m}$ and $v_{m}$ are given in the Appendix. When $m$ is associated with an asymmetric mode, we have

$$
\begin{aligned}
& \mu_{m}=\bar{\mu}_{m} \\
& n_{m}=0
\end{aligned}
$$

where $\bar{\mu}_{m}$ is given in the Appendix. The symmetric array $\varepsilon_{i j}$ is

$$
\varepsilon_{i j}=\int_{-1}^{1} \frac{\mathrm{d} Z_{i}}{\mathrm{~d} \Gamma} \frac{\mathrm{d} Z_{j}}{\mathrm{~d} \Gamma} \mathrm{d} \Gamma
$$

After introducing the mode shapes, the integrals in (29) are elementary and may be expressed in closed form. The results are omitted here for brevity.

For impulsive pressure imparting an initial radial velocity distribution, the initial conditions in dimensionless form are

$$
\begin{aligned}
& \zeta(\theta, 0)=0 \\
& \dot{\zeta}(\theta, 0)=\frac{v_{0}}{c} f(\Gamma)
\end{aligned}
$$

where $f(\Gamma)$ is a distribution function with value unity at $\Gamma$ equal to zero, i.e. at the center of the span. The actual velocity is $c \dot{\zeta}$. Introducing the representation (25) into (30), multiplying both sides by $Z_{n}$, and using the orthogonality condition (A10) yields

$$
\begin{aligned}
& T_{n}(0)=0 \\
& \dot{T}_{n}(0)=\frac{\varepsilon}{\pi^{2}} \int_{-1}^{1} f(\Gamma) Z_{n} \mathrm{~d} \Gamma
\end{aligned}
$$

where $\varepsilon$ is the velocity parameter

$$
\varepsilon=\varepsilon_{0} \pi^{2} / c \beta^{2}
$$

The total energy per unit length along the cylinder imparted to the shell is in dimensionless form

$$
\tilde{E}=\frac{\bar{T}(0)}{\beta \alpha^{2}}=\frac{1}{2} \frac{\lambda^{4} \tilde{\varepsilon}^{2}}{\pi^{4}} \int_{-1}^{1} f^{2}(\Gamma) \mathrm{d} \Gamma .
$$

The problem is now reduced to integrating (26) subject to the initial conditions (31). For a given distribution we will consider dynamic snap-through as a discontinuity in the response with respect to a change in $\varepsilon$. As shown by Hsu [1]. such a discontinuity cannot 
occur for any distribution if $\tilde{E}$ is less than $\tilde{V}_{c}$. For a fixed distribution, however, a discontinuity will not necessarily occur if this condition is violated. To determine the energy level associated with snap-through necessitates integration of the equations of motion.

We have considerable freedom in choosing a response parameter. A discontinuity in the motion with respect to initial conditions will be reflected in a number of measures. Because of its previous use in numerical studies of dynamic snap-through, we adopt here the response parameter

$$
\delta(\tau)=\frac{\int_{-1}^{1} a \zeta d \Gamma}{\int_{-1}^{1} H \mathrm{~d} \Gamma}=-3 \sum_{m} n_{m} T_{m} .
$$

It represents the ratio of the average radial displacement to the average rise of the shell.

\section{NUMERICAL RESULTS}

For most of the numerical work we restrict our attention to nearly symmetric distributions of the form

$$
f(\Gamma)=\cos \frac{\pi \Gamma}{2}+k \frac{3 \sqrt{ } 3}{2} \Gamma\left(1-\Gamma^{2}\right)
$$

in which $k$ is small compared to unity and represents the maximum value of an asymmetric deviation from the cosine distribution.

The nonlinear response was obtained by numerically integrating equation (26) on a digital computer using the Runge-Kuta method. At each time step the response parameter (34) was computed. As a check on the numerical accuracy, the total energy was monitored at each time step. It never varied from its initial value $\tilde{E}$ by more than 0.2 per cent and the variation was usually considerably smaller.

The time step size and the number of modes retained were empirically determined by examining the numerical convergence of the solution. It was found that a time step of onetwentieth of the half period corresponding to the highest linear mode shape retained is sufficiently small. The number of modes retained was such that retaining an additional mode changed the maximum value of $\delta$ occurring in the first half cycle by less than one per cent. The impulse parameter $\varepsilon$ was chosen such that $\delta$ exceeded unity for this determination. The results showed that the number of modes required increases with increasing $\lambda$, two modes being inadequate for all geometries considered. The indicated convergence was obtained using four modes for $\lambda \leq 3$, five for $\lambda \leq 5$, six for $\lambda \leq 7$ and eight for $\lambda \leq 10$.

We first consider symmetric snap-through by setting $k$ equal to zero in (35). For a given value of $\lambda$ the maximum value of $\delta$ which was attained during the first half cycle of motion was computed for various values of the velocity parameter $\varepsilon$. The results are shown in Fig. 4. For sufficiently small values of $\lambda, \delta_{\max }$ varies continuously with $\varepsilon$. For values of $\lambda$ greater than 2.8, however, there is a particular value of $\varepsilon$ at which the response shows a distinct jump, indicating dynamic snap-through. In Fig. 5 the energy level associated with the critical values of $\varepsilon$ is shown as a function of $\lambda$ Also shown is the stability sufficiency condition previously determined. As $\lambda$ increases it is an increasingly conservative estimate of the energy level required to produce immediate symmetric snap-through.

To further investigate the behavior of the nonlinear response, a number of computations were made at energy levels below that required for immediate dynamic snap-through. The 


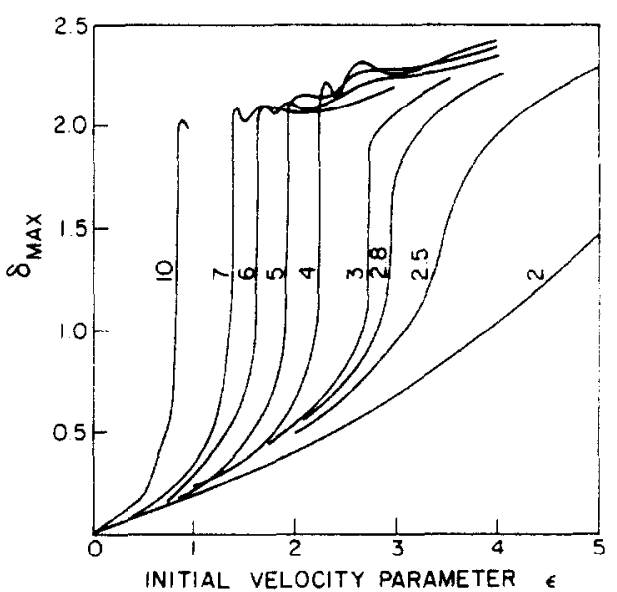

FIG. 4. Response parameter $\delta_{\max }$ vs. velocity parameter $\delta$

integration was carried out to $\tau$ equal to 100 , which for the values of $i$ considered is about 10-15 periods of the linear solution associated with the fundamental mode shape. The results are shown in Figs. 6-10. For comparison the constant values of $\dot{\delta}$ computed for the first two symmetric and the first nonsymmetric critical configurations are shown in the figures.

The solid curves in Fig. 6 are the responses for $\lambda$ equal to six and $\varepsilon$ values of 1.1 and 1.2. The critical value of $\varepsilon$ for immediate snap-through is about 1.6. For both cases the first maximum of $\delta$ is considerably less than unity. For $\varepsilon$ equal to $1 \cdot 1$ this remains true for all of the relative maximums of $\delta$ for the time period examined. For the slightly higher value of

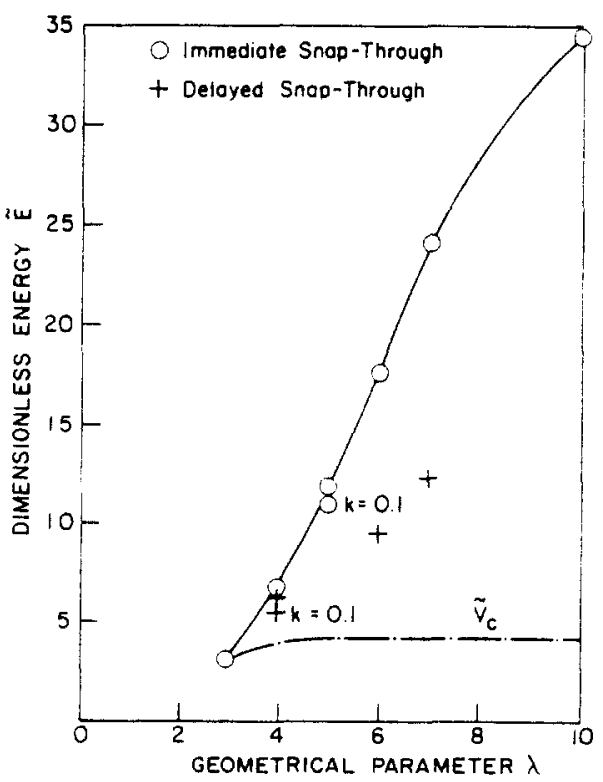

Fic. 5. Energy levels for snap-through vs. ; 


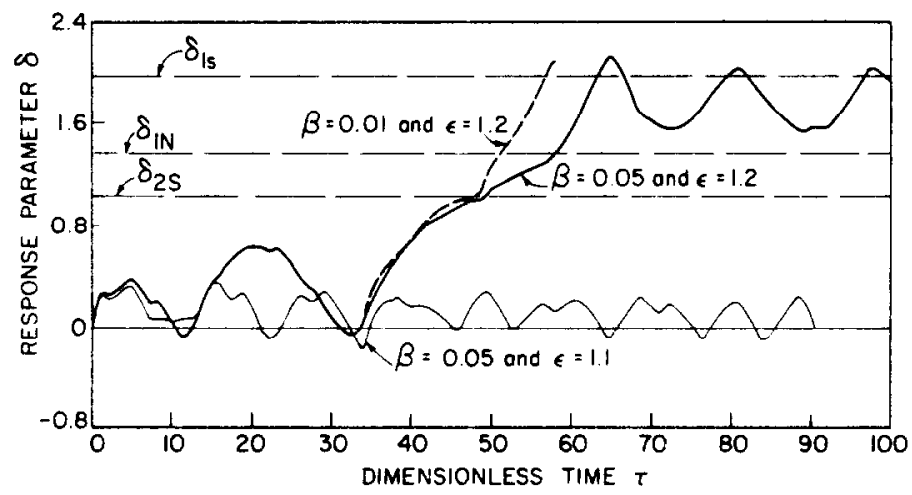

F1G. 6. Response curves for $\lambda=6$.

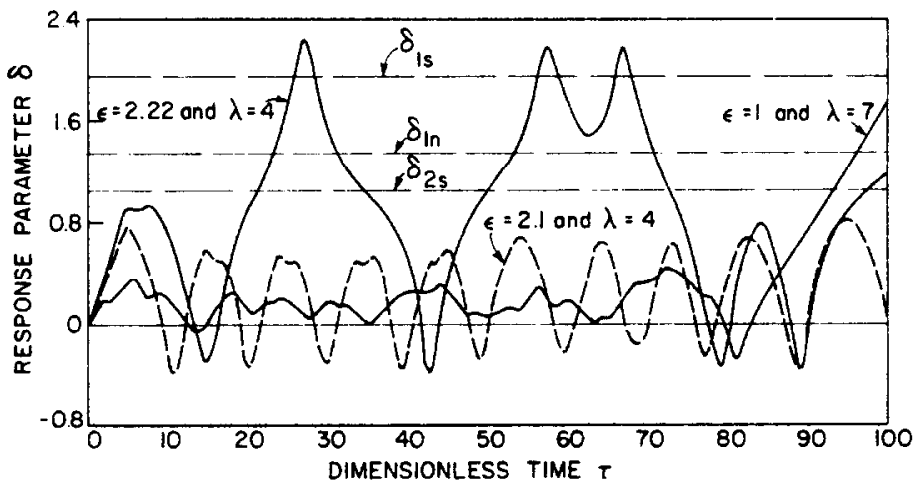

FiG. 7. Response curves for $\lambda=4$ and $\lambda=7$.

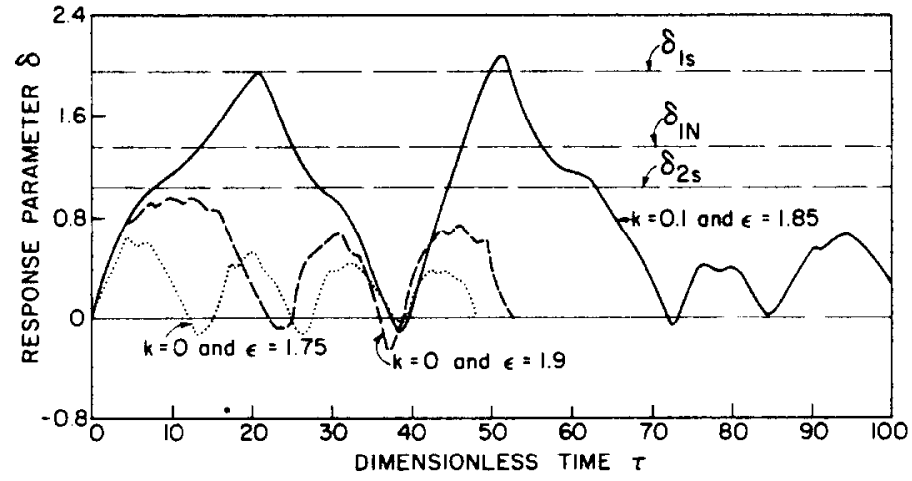

FIG. 8. Response curves for $i=5$. 


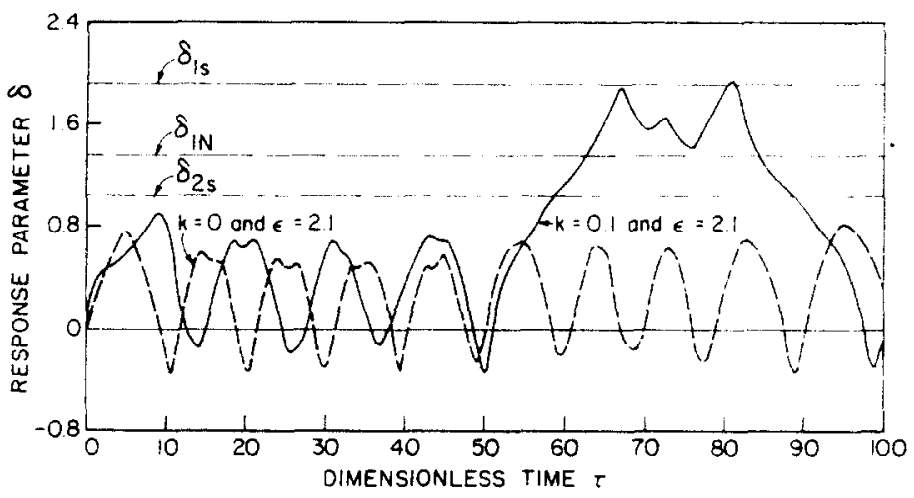

FIG. 9. Response curves for $i=4$.

$1 \cdot 2$, however, the result is much different. After two oscillations with $\delta$ less than unity, the shell snaps through. This phenomenon of delayed snap-through was observed in a number of cases, but the detailed behavior varied considerably with $\lambda$. In Fig. 7 delayed snapthrough was obtained for $\lambda$ equal to seven at $\varepsilon$ equal to 1.0 . The jump occurred after several oscillations. In contrast for $\lambda$ equal to four the jump first occurred on the second oscillation.

The energy levels of the responses exhibiting delayed snap-through are shown as points in Fig. 5. It is difficult to obtain a critical energy curve for delayed snap-through. Extensive numerical computation is required. Moreover there is no a priori condition for determining the time interval that should be examined. Nevertheless the three points in Fig. 5 suggest that a critical curve could be obtained. This curve would not, however, distinguish between the elapsed times required for snap-through to occur. If the elapsed time is too large, of course, the phenomenon loses its physical significance in the presence of damping.

One other point needs to be considered in connection with delayed snap-through. Examination of (26), (31) and (34) shows that $\delta$ as a function of $\varepsilon$ does not explicitly depend upon the semi-opening angle $\beta$. The only influence of $\beta$ is through the quantities $n_{m}$ and $s_{i j}$ which arise from integration of the linear mode shapes. The results here have been obtained for $\beta$ equal to 0.05 . As shown in the Appendix, however, the mode shapes are very insensitive to $\beta$, and the values of $n_{m}$ and $\varepsilon_{i j}$ for other values of $\beta$ in the range of interest would differ by the order of 1 per cent. It seems reasonable, then, that the critical values for snap-through

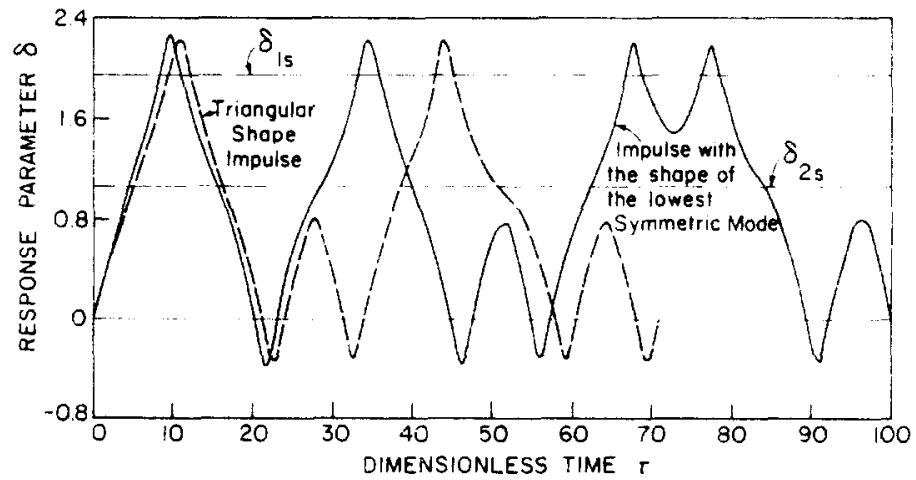

FIs. 10. Response curves tor $i=4$ for different distributions. 
would be independent of $\beta$. This is in fact true for immediate snap-through. But the dashed curve in Fig. 6 shows one response for $\beta$ equal to 0.01 which deviates from the response for $\beta$ equal to 0.05 for times exceeding 50 . One possible explanation is the nonlinear coupling which permits energy transfer to modes which are not initially excited. The rate of growth of these modes depend. of course, upon the coefficients in the system of equations. Small changes in the growth rate would eventually manifest themselves after sufficient elapsed time. The extensive numerical computation required prohibited a detailed investigation of the effect of $\beta$ on the long term motion. It is not expected that such an investigation would make any qualitative change. but the above remarks suggest that the quantitative values for the occurrence of delayed snap-through might be affected.

To investigate the effect of small deviations from symmetry, a few computations were made using the distribution (35) with $k$ equal to $0 \cdot 1$. In general the presence of an asymmetric component enhances the possibility of snap-through. Response curves for $\lambda$ equal to five are shown in Fig. 8 for three values of $\varepsilon$, all of which are below the critical value for immediate symmetric snap-through. For the two cases with $k$ equal to zero, snap-through has not occurred. But with an asymmetric component, immediate snap-through occurs at an intermediate value of $\varepsilon$. Figure 9 demonstrates a similar influence on delayed snap-through. The reduction in initial energy required to cause snap-through when $k$ is equal to 0.1 is shown for these two cases in Fig. 5.

Finally Fig. 10 shows the response for two symmetric distributions in the form of a triangular shape and the shape of the lowest symmetric mode. The initial energy for the cases shown is the same as for the response to the cosine distribution for $\lambda$ equal to 4 shown in Fig. 7. For this energy level the response to the cosine distribution exhibits delayed snapthrough, whereas in Fig. 10 immediate snap-through has clearly occurred. A detailed investigation of these distributions has not bein made, but this example indicates that the critical energy level is sensitive to the shape of the symmetric distribution as well as to the presence of asymmetric components.

\section{DISCUSSION}

The critical configurations have an important role in determining the type of motion that the shell may exhibit. We have used the critical configurations for determining a general sufficiency condition. When specific examples are considered, the numerical results have shown that a wide variety of dynamic behavior may be exhibited. For sufficiently high impulsive loading the shell will undergo immediate snap-through. It may also exhibit delayed snap-through after varying periods of elapsed time at significantly lower levels of loading. In addition the occurrence of snap-through is sensitive to changes in shape of the symmetric distribution as well as small deviations from symmetry.

Of course the energy levels associated with the various types of snap-through all exceed the sufficiency condition. In fact the latter is quite conservative for a substantial range of the shallowness parameter $\lambda$. Moreover the stability criterion obtained for a specific case may bear little resemblance to the sufficiency condition.

The following heuristic argument is an attempt to give a general interpretation to these results. As a motion is traced out in functional configuration space, a corresponding surface path is traced out on the potential energy surface. The general features of this surface are determined by the critical configurations In a generalized sense. stable equilibrium 
configurations are the low points of valleys in the surface. Unstable configurations will occur at the high point of a hill or as a pass or saddle point in the ridges separating different valleys.

In the present problem only one critical configuration is stable in addition to the undeformed state. This is the symmetric configuration with the lowest energy level and represents a stable snapped-through state. All remaining configurations are unstable and necessarily lie on the ridge system separating the two valleys around the stable undeformed and snapped-through configuration points.

For impulsive loading of an undeformed shell, all surface paths originate from the bottom of the valley around the undeformed configuration point. These paths can only lead out of this valley through passes or over ridges. If the tangent to the surface $p$ th has just the right direction at the origin (i.e. the right distribution of initial velocity), the path may exit the valley directly through a pass with the energy level of the critical configuration which is located at the pass. On the other hand, if the direction of the tangent is held constant (i.e. the distribution of the initial velocity is prescribed), the surface path during the early stage of motion may not come near any critical point. Thus immediate snap-through can occur only if the initial kinetic energy is sufficient for the surface path to reach the top of a ridge.

If the modes were uncoupled, a response with energy insufficient for immediate exit would be an oscillatory motion tracing out the same surface path. For our problem. however, the nonlinear coupling may result in the parametric excitation of modes which are not initially excited. In this case the surface path will be a complicated curve traced out over the valley, permitting the possibility of encountering a lower ridge. Such an encounter would be manifest as a delayed snap-through of the shell.

The lowest pass out of the valley around the undeformed configuration point is, for $;$ greater than $\tilde{\lambda}_{1}$, the nonsymmetric configuration with lowest energy level. The subspace of purely symmetric motion does not include this point, and the energy level required to exit the valley in this subspace is necessarily higher. Since the energy levels of all symmetric configurations in the ridge system are greater than the lowest pass. the ridge must "slope downward" in some direction as we move from the symmetric subspace. Thus even small deviations from symmetry may result in the surface path encountering a lower ridge. with a consequent reduction in the initial energy required for snap-through.

It is clear that the rather high energy levels required for snap-through in the problem considered here is due to the form of the initial velocity distribution. A distribution with large asymmetric components would snap-through at substantially lower values. Even for purely symmetric distributions a wide variation is possible. Comparing the energy level found for immediate snap-through for the cosine distribution with the energy levels of the various symmetric configurations in Fig. 2 shows that for most of the range of $i$ there are configurations with both higher and lower energy levels. Thus it must be possible to pick a distribution giving a surface path which would cross the ridge at either a higher or lower energy level than that for the cosine distribution.

Thus the energy levels required for dynamic snap-through will vary markedly for different distributions of initial velocity. These levels may be significantly different than the sufficiency condition. The results indicate, however, that a criterion for a specific distribution must be viewed with some caution. If the distribution is such that immediate snap-through occurs when the surface path associated with the motion crosses a relatively high point on the ridge, two results can be anticipated. The energy level will be sensitive to small deviations in the distribution, and the shell will exhibit delayed snap-through. 
Finally we consider the implication of truncating the modal representation to $N$ terms. We are attempting to span the functional displacement space with a $N$-dimensional coefficient vector. We are, in effect, constraining the motion to paths for which all coefficients with index greater than $N$ vanish. It is difficult to say how constrained and unconstrained paths of impending snap-through would differ. We know, however, that the significant topological features of the functional space are determined by the critical configurations. Thus it is necessary that the $N$-dimensional subspace include the critical configurations, i.e. that the critical configurations are accurately represented by a modal expansion truncated to $N$ terms.

In the present problem this was used to check the number of modes empirically determined for the case $\lambda$ equal to five. A six term modal representation was used to compute the strain energy associated with the critical configurations. The results agreed to within 1 per cent with the values obtained from (22).

Acknowledgement-This work was supported by the National Science Foundation through Grant No. GK-10725.

\section{REFERENCES}

[1] C. S. Hsu, On dynamic stability of elastic bodies with prescribed initial conditions. Int. J. Engng Sci. 4. I (1966).

[2] I. K. MCIvor and C. H. Popelar. Dynamic stability of a shallow cylindrical shell. J. Engng Mech. Dir. Am. Soc. Cit. Engrs 93. No. EM 3 (1967).

[3] C. S. Hst. The effects of various parameters on the dynamic stability of a shallow arch. J. appl. Mech. 34 (1967).

[4] C. S. Hst. Stability of shallow arches against snap-through under time wise step loads. J. appl. Mech. 35 (1968).

[5] C. S. Hsi. Equilibrium configurations of a shallow arch of arbitrary shape and their dynamic stability character. Int. J. Non-linear Mech. 3 (1968).

[6] N. C. Huang and W. NachBar. Dynamic snap-through of imperfect viscoelastic shallow arches. $J$. appl. Mech. 35 (1968).

[7] L. J. OvensHiRE. Dynamic Snap-through Stability of the Nonlinear Elastic Plane Strain Free Vibration of a Shallow Cylindrical Shell. Ph.D. Dissertation. University of Michigan (1969).

[8] E. Reissner. On transverse vibrations of thin, shallow elastic shells. Q. appl. Math. 13 (1955).

\section{APPENDIX}

\section{Infinitesimal vibrations}

Neglecting tangential inertia and discarding all second order terms reduccs the lincar free vibration problem to solving the governing equation

$$
\check{\zeta}^{\prime \prime}+\alpha^{2}\left(\zeta^{\prime \prime \prime \prime}+2 \zeta^{\prime \prime \prime}+\zeta^{\prime \prime}\right)=0 .
$$

The solution for general boundary conditions and arbitrary initial conditions is discussed in detail in Ref. [7]. For the simple support boundary conditions (10), the solution is

$$
\left.\zeta=\sum_{n}\left[\bar{C}_{n} \sin \left(\bar{\mu}_{n} \tau+\bar{\phi}_{n}\right) \bar{Z}_{n}(\theta)+\bar{C}_{n} \sin \left(\tilde{\mu}_{n} \tau\right)+\tilde{\phi}_{n}\right) \tilde{Z}_{n}(\theta)\right]
$$

where the bar denotes an asymmetric mode and the tilde denotes a symmetric mode. The quantities $C_{n}$ and $\phi_{n}$ are arbitrary constants determined from the initial conditions. 
The dimensionless circular frequencies are

$$
\begin{aligned}
& \tilde{\mu}_{n}=\left(n^{2} \pi^{2}-\beta^{2}\right) / \lambda^{2} \\
& \tilde{\mu}_{n}=\left(v_{n}^{2}-\beta^{2}\right) / \lambda^{2}
\end{aligned}
$$

where $v_{n}$ is a root of the characteristic equation

$$
\tan v=-\frac{v^{3} \tanh w}{u^{3}}+\frac{2 u^{2} v}{w^{2}}\left(1-\frac{v^{2} w^{2}}{\lambda^{4}}\right)
$$

in which

$$
\begin{aligned}
& w^{2}=v^{2}-2 \beta^{2} \\
& u^{2}=v^{2}-\beta^{2}
\end{aligned}
$$

The asymmetric mode shapes are

$$
\bar{Z}_{n}=\sin n \pi \Gamma
$$

in which

$$
\Gamma=\theta / \beta
$$

The symmetric mode shapes are

$$
\tilde{Z}_{n}=A_{n}\left(\cos v_{n} \Gamma+\frac{v_{n}^{2}}{w_{n}^{2}} \cos v_{n} \frac{\cosh w_{n} \Gamma}{\cosh w_{n}}-\frac{2 u_{n}^{2}}{w_{n}^{2}} \cos v_{n}\right)
$$

where

$$
\begin{aligned}
A_{n}= & \left\{1+\frac{\sin 2 v_{n}}{2 v_{n}}+\frac{v_{n}^{4} \cos ^{2} v_{n}}{w_{n}^{4} \cosh ^{2} w_{n}}\left(\frac{\sinh 2 w_{n}}{2 w_{n}}+1\right)+\frac{8 u_{n}^{4}}{w_{n}^{4}} \cos ^{2} v_{n}\right. \\
& +\frac{2 v_{n}^{2} \cos v_{n}}{w_{n}^{2} \cosh w_{n}}\left[\frac{w_{n} \sinh w_{n} \cos v_{n}+v_{n} \cosh w_{n} \sin v_{n}}{u_{n}^{2}}\right] \\
& \left.-\frac{4 u_{n}^{2} \sin 2 v_{n}}{w_{n}^{2} v_{n}}-8 \frac{v_{n}^{2} u_{n}^{2} \cos ^{2} v_{n} \tanh w_{n}}{w_{n}^{5}}\right\}^{-!} .
\end{aligned}
$$

The mode shapes satisfy the orthogonality condition

$$
\int_{-1}^{1} Z_{m} Z_{n} \mathrm{~d} \Gamma=\delta_{m n}
$$

where $\delta_{m n}$ is the Kronecker delta and $Z_{m}$ represents either a symmetric or an asymmetric mode.

It is worth noting that in (A3) and (A5) the term $\beta^{2}$ is small compared to the first term. Thus the dimensionless circular frequencies and mode shapes are essentially independent of $\beta$. depending only upon the geometric parameter $\lambda$. Also the higher roots of (A4) approach odd multiples of $(\pi / 2)$. With this the symmetric mode shapes approach

$$
Z_{n}=\cos \frac{\left(2_{n}+1\right)}{2} \pi \Gamma \text {. }
$$


The value of $n$ for which (A11) is valid. however. depends upon $\lambda$. For example for $\lambda$ equal to three. the approximation is valid for $n$ greater than two: whereas for i equal to ten, $n$ must be greater than five.

(Received 22 Mav 1970: rerised 24 August 1970)

\begin{abstract}
Абстракт-Используется теория пологой оболочки для исследования нелинейного поведения чилиндрической панели, подверженной плоскому движению. которое является реакиией на симметрическое импульсивное давление, вызываюшее начальное распределение скорости для оболочки. Получается достаточное условие для устойчивости, рассматривая критическую или равновеснчю конфигурацию. Получается критическое значение, которое вызывает внезапное динамическое прошелкивание для косинусного распределения. Для большинства геометрий достатопным условием яаляется действите.льно консервативная оценка критического значения. Это значение очень чуствительно на малые отклонения от симметрии. Оболочка может также вызывать замедляющее прощелкивание для значительно низких значений начальной скорости. Однако, для этих двух случаев достаточным условием остается консервативная оценка. Она выводится из кауественного топологического аргумента. что отмеченно разные результаты можно было получить для упругих распределений.
\end{abstract}

